CDD: 412

\title{
ACCIÓN, HECHO Y SUCESSO ${ }^{1}$
}

\author{
TOMÁS BARRERO \\ Departamento de Filosofia \\ Universidad de los Andes \\ COLOMBIA
}

ta.barrero32@uniandes.edu.co

Received: 16.03.2011; Revised: 04.07.2012; Accepted: 10.07.2012

\begin{abstract}
Examino la semántica de las oraciones de acción de Davidson y sus aspectos más relevantes: la formalización cuantificacional y las tesis sobre preposiciones y sobre modificación adverbial. A partir de Grice, emprendo tres líneas de ataque contra esta propuesta: una mezclando predicados y preposiciones, otra basada en modificaciones adverbiales y una a partir del posible análisis acciones negativas. Con ello espero suscitar dudas acerca del papel de los hechos y sucesos en el análisis de la acción.
\end{abstract}

Keywords: Forma lógica y lenguaje natural; Acciones, hechos y sucesos; Consecuencia lógica y análisis dimensional.

Abstract: In this paper I asses Davidson's analysis of action sentences stressing its quantificational structure and its views on prepositions and adverbial modification. Three arguments inspired by Grice's ideas are deployed against it. First, I point to weird consequences of taking prepositions as defining predicates; second, I show some obstacles for Davidson's view on adverbs; finally, I raise some doubts about his putative analysis of "negative actions". From these, I hope that connections among actions, facts and events should be reconsidered in action theory.

\footnotetext{
${ }^{1}$ Este trabajo hace parte del proyecto de investigación "Lenguaje y acción. Fundamentos de una semántica multidimensional" financiado por la Universidad de los Andes. En sus diferentes versiones recibió valiosos comentarios de David Rey, Carlos Márquez, Douglas Niño, Mauricio Rengifo, Carlos Cardona, Raúl Meléndez, Lina Trigos y Carlos Patarroyo. A todos ellos mi gratitud; los errores restantes son míos.
}

Manuscrito - Rev. Int. Fil., Campinas, v. 35, n. 2, p. 207-231, jul.-dez. 2012. 
Keywords: Logical Form and natural language. Actions, facts and events. Logical consequence and dimensional analysis.

\section{INTRODUCCIÓN}

Las oraciones de acción son constituyentes simples del lenguaje natural que, sin embargo, han motivado reacciones teóricas muy diversas. Desde la semántica formal a la teoría de actos de habla, hay una densa línea de discusión cuyas ramificaciones exceden los límites de mi trabajo. En él voy a partir de la posición de Davidson con respecto a la forma lógica de las oraciones de acción. Discutiré su tesis de que las oraciones de acción son completamente analizables en una forma lógica de primer orden que incluya cuantificaciones sobre clases de sucesos individuales (i.e. sobre hechos). Contrastaré la estrategia de Davidson con otra basada en la idea de "infortunios" y que denomino "multidimensional" usando tres ejemplos: los verbos intransitivos con preposiciones, la condiciones de aplicación de ciertos adverbios y las acciones negativas, aquellas que consisten en hacer que algo no suceda. Aunque el énfasis de mis objeciones es semántico, varias consecuencias generales afectan a la ontología de la acción tal como la concibe no sólo Davidson, sino buena parte de la tradición analítica. Reconozco, sin embargo, que mis tres críticas a la primera propuesta son insuficientes para descartarla y sustituirla por una opción más plausible. Mi ejercicio en la sección 2 puede verse como la versión preliminar y programática de un proyecto de trabajo encaminado a construir un argumento crítico, riguroso y de mayor alcance en otros trabajos ya en desarrollo Barrero (2011a, 2011b). 


\section{LA ESTRATEGIA DE LA CONSECUENCIA LÓGICA}

"La estrategia de la consecuencia lógica" puede describirse brevemente así: las propiedades semánticas de las oraciones de acción pueden establecerse con una formalización que establezca sus consecuencias lógicas. La dificultad de los aspectos involucrados en esa formalización ha generado una multiplicidad de posiciones en semántica formal. Las más importantes responden a dos tradiciones semánticas. Inspirados por la idea de proposición de Russell, Barwise y Perry (1981) hacen de las situaciones abstractas los primitivos semánticos y les atribuyen como elementos constitutivos el tiempo verbal y el espacio. Otras posiciones provienen de Tarski, por ejemplo la de Montague (1969) basada en teoría de modelos y en la idea de proposición de Frege y la de Davidson (1967) en términos de condiciones de verdad. Ambas argumentan a favor de la necesidad de introducir sucesos en la ontología para darle sentido a una buena porción del discurso de la psicología o de la ética. Montague prefiere un enfoque intensional de las proposiciones y una cuantificación de segundo orden para identificar los sucesos denotados por ellas a partir de predicados temporales; Davidson, con una lógica extensional y de primer orden, muestra que las consecuencias lógicas intuitivas de las oraciones de acción exigen contar con sucesos.

Cada una de estas decisiones metodológicas involucra una postura con respecto a la ontología. Por ejemplo, que hay combinaciones de objetos que incluyen vectores espacio-temporales, o bien que hay objetos del tipo proposición y se puede cuantificar sobre ellos, o bien que, además de casas, personas y números, hay cosas como asesinatos, saltos y caídas. En lo que sigue me concentraré en la tercera opción. Abordaré la propuesta de Davidson desde tres rasgos que considero determinantes y a la vez enigmáticos: sus compromisos ontológicos, su lectura de las preposiciones y algunas de sus tesis sobre modificación adverbial. 


\subsection{Forma lógica y compromisos ontológicos}

La sutileza y originalidad de la propuesta de Davidson se aprecian mejor cuando se la compara con el análisis gramatical corriente de una oración de acción. Según este último, normalmente tendremos dos sintagmas, uno nominal y otro verbal y en el nominal encontraremos un nombre o expresión referencial - probablemente con modificadoresy en el verbal un verbo conjugado, los complementos de objeto directo o indirecto $-\mathrm{o}$ los dos- y complementos circunstanciales. De acuerdo con esa idea y usando la lógica de primer orden, la forma lógica de:

1.1. Mi vecino mató a mi burro

es

1.2. $\exists x \exists y[\operatorname{Mi} \operatorname{vecino}(x) \wedge \operatorname{Mi} \operatorname{burro}(y) \wedge$ Mató a $(x, y)]$.

Dado que 1.2 es una fórmula corriente, las oraciones de acción no se diferencian lógicamente -i,e. semánticamente hablando, por sus consecuencias lógicas - de las otras oraciones.

Davidson desafía esta opinión recibida. Su tesis sobre la forma lógica es una declaración de principios acerca de cómo tratar el lenguaje natural (1967, p. 174):

La forma lógica se inventó para hacer un contraste con otra cosa que está a la vista pero que es un mero espejismo, esto es, la forma que nos sentimos llevados a asignar a las oraciones por una analogía superficial o por la gramática tradicional. Lo que el ojo o el oído encuentran en el lenguaje tiene el encanto, la complejidad, la comodidad y el engaño de otras convenciones en uso; pero, por debajo de eso, está la moneda sólida de una estructura más sencilla y adusta, sin brillo, pero también sin engaño. Esta moneda auténtica, la estructura profunda, no necesita 
mostrarse directamente en las transacciones de la vida real. En la medida en que sepamos cómo hacer efectivo un documento podremos gozar de los beneficios del crédito.

El significado de una oración (que se consigue a partir de sus condiciones de verdad) se da en términos del papel que jueguen sus componentes en dicha estructura. Dado que conocer el significado de una oración de acción implica conocer sus consecuencias lógicas, aprender a usar un verbo implica saber cuántas variables y cuántos modificadores admite en virtud de su forma lógica. Por otra parte, involucra saber qué verbos están ligados con la introducción de agentes en tanto así lo requieren las consecuencias lógicas de las oraciones en las que aparecen. La principal innovación de Davidson es conectar el análisis de una oración de acción con el análisis de una oración indicativa corriente. Allí donde aparezca una expresión referencial en una, aparecerá una variable para suceso en la otra; allí donde adjetivos atributivos en ésta, adverbios atributivos en aquella. El paralelo es limitado porque, mientras los adjetivos atributivos nos comprometen directamente con cierta propiedad predicada de un objeto, los adverbios atributivos muchas veces requieren la introducción de un agente para producir las consecuencias lógicas esperadas. Por ejemplo, el análisis de

1.3. "Mi vecino mató a mi burro inmediatamente de un tiro en la frente"

implica que mi vecino mató a mi burro y que lo mató con un tiro en la frente. Pero el tránsito de $\mathbf{1 . 3}$ a que el tiro de mi vecino lo mató inmediatamente requiere una descripción de la acción que permita vincular en un mismo hecho a mi burro y a mi vecino. Este hecho y el papel referencial que tienen algunos pronombres en función anafórica en el discurso de acción ("lo", "eso", etc.) llevan a Davidson a introducir una cuantificación existencial con la que se expresa el hecho y 
sus características. De acuerdo con eso las siguientes fórmulas deben seguirse de la forma lógica de $\mathbf{1 . 3}$ :

1.4. $\exists x[$ Mató a(Mi vecino, Mi burro, $x)]$, en adelante $\psi$,

1.5. $\exists x[$ Con un tiro en la frente $(x)]$,

\section{y}

1.6. $\exists x[$ De inmediato $(x)]$.

Luego, la forma lógica de $\mathbf{1 . 3}$ es:

1.7. $\exists x[$ Mató a(Mi vecino, Mi burro, $x) \wedge$ Con un tiro en la frente $(x) \wedge$ De inmediato $(x)$ ], en adelante $\varphi$.

La cuantificación existencial exige condiciones de identidad y, por ende, saber a qué se refieren las variables cuantificadas. Por analogía con los adjetivos atributivos, diferenciamos el hecho — "fact"- como cualquier estado de cosas en el que se presentan las condiciones para que la oración de acción y la predicación de un adjetivo atributivo sean verdaderas, del suceso — "event" - como aquella entidad particular de la que se predican los modificadores adverbiales y el individuo del que se predica la propiedad expresada por el adjetivo atributivo, respectivamente. El primero, pero no el segundo, se obtiene por generalización sobre las secuencias de objetos que satisfacen la oración; el segundo, pero no el primero, establece una correlación entre variables y objetos mediante la función de interpretación a partir de la cual se construye la idea misma de satisfacción. Tal como no decimos de un individuo que sea verdadero, no decimos de un suceso que sea verdadero; así como decimos que para que algunas oraciones con adjetivos atributivos puedan ser verdaderas tiene que haber individuos, decimos que para que las oraciones de acción puedan ser verdaderas tiene que haber sucesos. La pregunta por los hechos es general y corresponde a una teoría de la 
verdad, la pregunta por los sucesos es particular y hace parte de una teoría de la referencia; aunque, si hemos de creerle a Davidson (1977), la segunda teoría es una parte propia de la primera. La conclusión de Davidson parece clara: las oraciones de acción expresan hechos y sólo mediante esa expresión se refieren a sucesos (1967, pp. 170-173)².

\subsection{Verbos y preposiciones}

Otra consecuencia de este análisis es la posibilidad lógica de separar las preposiciones de los verbos. El caso más claro y curioso es el de los verbos intransitivos. Por ejemplo el análisis de:

1.8. "Colón llegó a América",

de acuerdo con Davidson debe ser:

1.9. $\exists x[$ Llegó(Colón, $x) \wedge \mathrm{A}($ América, $x)]$,

que implica lógicamente

1.10. $\exists x[\mathrm{~A}($ América, $x)]$.

\footnotetext{
${ }^{2}$ Obsérvese que esta formulación no padece prima facie de los defectos de las teorías de la correspondencia más corrientes porque asume una posición tarskiana que no requiere la referencia a hechos. Mientras la referencia a hechos unifica el camino por el que llegamos a la verdad de una oración, la semántica tarskiana permite una pluralidad de caminos por los cuales se produce la generalización de satisfacción a verdad. Aparentemente el camino en las oraciones de acción es justamente la generalización de sucesos a hechos. En segundo lugar, el papel que Davidson les atribuye a los hechos y sucesos en el análisis de las oraciones de acción es fundamentalmente interpretativo: los hechos y sucesos son entidades teóricas cuya existencia garantiza que se dan las consecuencias de las oraciones de acción para que podamos comprender el significado de los verbos en dichas oraciones. Para una discusión de esta versión tarskiana y su relación con la correspondencia Davidson (1969, pp. 66-67). Para una exposición propiamente tarskiana Tarski (1944, pp. 289291).
} 
Quiero señalar la asimetría entre 1.4 y 1.10: mientras en el primer caso tenemos un predicado estructurado que incluye una preposición, en el segundo hay una preposición que permite definir un predicado. En términos de la propuesta de Davidson, dicho predicado no tiene nada de particular y su rango de aplicación es un conjunto de sucesos.

\subsection{Modificación adverbial}

El último rasgo que quisiera resaltar tiene que ver con las particularidades de la modificación adverbial. La propuesta implica la existencia de adverbios "atributivos" que califican sucesos, como $\mathbf{1 . 6}$ y complementos circunstanciales que pueden cumplir con esa misma función, como 1.5. El caso difícil es el de "adverbios de acción" "intencionalmente", "voluntariamente" - porque, dada la tesis de que la ontología de la acción es común a la ciencia, hay que eliminar la posibilidad de que existan rasgos de la acción que no puedan ser explicados en términos extensionales ${ }^{3}$. Si dichos adverbios fueran atributivos éste sería el caso y la explicación de la acción no sería uniforme con respecto a la explicación de otros sucesos. Pero esta implicación dualista es intolerable para Davidson (1995b, p. 6). Luego, la intencionalidad, por ejemplo, no es un rasgo de la acción sino una forma posible de describirla Davidson (1974). De acuerdo con esta lectura, la forma lógica de:

1.11. "Mi vecino mató intencionalmente a mi burro inmediatamente de un tiro en la frente",

es

1.12. Fue intencional por parte de mi vecino que $(\varphi)$,

${ }^{3}$ Discuto en detalle otros problemas conectados con la acción intencional en Barrero (2010).

Manuscrito - Rev. Int. Fil., Campinas, v. 35, n. 2, p. 207-231, jul.-dez. 2012. 
donde "Fue intencional por parte de mi vecino que" es una función oracional análoga a los operadores modales o epistémicos y no un predicado. La negación de $\mathbf{1 . 1 2}$ tiene las propiedades usuales: hace parte de su mismo espacio lógico y eso quiere decir que ambas fórmulas, afirmación y negación, se refieren al mismo suceso y expresan hechos incompatibles.

\section{LA ESTRATEGIA MULTIDIMENSIONAL}

El principio de la sofisticada propuesta davidsoniana es simple: la consecuencia lógica nos da todo lo que basta para una semántica comprehensiva de las oraciones de acción. En esta sección desarrollaré tres argumentos para mostrar que la estrategia de la consecuencia lógica es restrictiva. Estas críticas nos hacen pensar la posibilidad de repensar críticamente la conexión entre acción, hechos y sucesos y reconsiderar la estrategia metodológica. La consecuencia lógica no es más que una de las múltiples herramientas para tratar el lenguaje natural. "Presuposición", "nulidad", "infortunio", por ejemplo, son candidatos igualmente competentes para esa tarea. Aunque reconozco que mis argumentos no son tan detallados como para descartar la propuesta de Davidson, espero que sean suficientes para generar sospechas sobre su coherencia y poder explicativo.

\subsection{Predicación y preposiciones}

Una seria limitación de la teoría de Davidson surge con las preposiciones $^{4}$, porque me parece claro que $\mathbf{1 . 1 0}$ involucra un predicado mal formado sintácticamente $\mathrm{y}$, por ende, no puede expresar una proposición ni una proposición completa o, en términos davidsonianos, no tiene

\footnotetext{
${ }^{4}$ En este punto y en la primera parte de la siguiente sección me inspiro en la crítica formulada por Grice (1986).
} 
condiciones de verdad. En efecto, se supone que un predicado bien definido tiene unas condiciones de aplicabilidad dadas. Pero

$$
\text { "A(América, } x) \text { " }
$$

no tiene un rango de aplicación determinado. Compárese con

$$
\text { "Par }(x) . "
$$

Mientras el primer caso requiere un tipo de compleción, el segundo no; es contextualmente obvio que en éste estamos hablando de números, pero ¿de qué estamos hablando con aquel? Podríamos parafrasearlo como " $x$ es un suceso a América", pero no parece una opción viable. Davidson propone algo como: "hubo un suceso que incluye movimiento a América" (1967, p. 160). Entonces el predicado es elíptico, incompleto y la forma de completarlo requiere que restrinjamos su aplicación a cierta clase de sucesos, los movimientos. La pregunta obvia es qué se mueve, o bien cómo podemos parafrasear las condiciones de aplicabilidad sin hacer referencia al objeto en movimiento. ¿Es posible que "movimiento" sea un primitivo semántico, por ejemplo en una teoría física alternativa? Supongamos que lo sea, ¿es un primitivo semántico de las oraciones de acción? Una oración de acción involucra un agente y a veces movimiento de ese agente, pero creo que es un error pensar que la descripción del movimiento tenga un significado independiente de la referencia al agente, como parece afirmarlo Davidson. Los agentes permiten definir el movimiento y así la acción, pero la relación es asimétrica. La forma lógica de Davidson en este caso no tiene una interpretación intuitivamente aceptable sin recurrir a una paráfrasis que nos compromete con los agentes y los verbos para comprender los movimientos.

Pero si éste es el método de compleción, se siguen dos consecuencias, en mi opinión contrarias a la propuesta general: los predicados con preposiciones son incompletos y su compleción requiere conocer el verbo al cual se vincula la preposición (en este caso "llegar"). Luego, los 
componentes de la fórmula $\mathbf{1 . 9}$ no se encuentran en el mismo nivel lógico. Mientras "Llegó(Colón, $x$ )" determina las condiciones de aplicabilidad de "A(América, $x$ )", la inversa no se da ${ }^{5}$.

Pero esto no es todo. Este resultado implica que la predicación falla por otra razón. Las condiciones de identidad implícitas en cualquier cuantificación existencial son relativas al verbo al que se le adjunta la preposición. Por ejemplo, sabemos que $\mathbf{1 . 1 0}$ es lógicamente equivalente a:

2.1. $\exists x(x=a) \wedge \mathrm{A}($ América, $a)$.

Por el argumento anterior, no podemos establecer a qué dominio de interpretación pertenece $a$ a no ser que recurramos a Llegó(Colón, $x$ ). Es decir, las condiciones de predicación de $\mathbf{2 . 1}$ requieren que

2.2. $\exists y(y=c) \wedge$ Llegó $(c, x)$,

se verifique. Luego, en este contexto la cuantificación sobre personas es más primitiva que la cuantificación sobre sucesos porque la primera permite definir la segunda a través de las condiciones de identidad, pero no al contrario ${ }^{6}$. Los predicados con preposiciones no permiten

\footnotetext{
${ }^{5}$ Puede haber otra explicación para el comportamiento anómalo de estos predicados: la dirección de los movimientos puede interpretarse como una característica espacial del hecho completo (Montague (1969)). Hay movimientos de Colón y algunos de ellos son en dirección a América; otros tienen lugar en 1492; otros son desde América; etc. Si ese es el caso, las preposiciones se deberían interpretar como predicados de segundo orden, lo que daría razón de las dificultades en los niveles de significado involucrados. Davidson estaría intentando expresar una propiedad de estados de cosas como si fuera un estado de cosas. He desarrollado esta idea en Barrero (2011a).

${ }^{6}$ Algunas posiciones inspiradas por Davidson concenden que la cuantificación sobre eventos contiene una cuantificación - implícita - sobre personas u objetos para obtener una forma lógica general de las oraciones de acción (Parsons (1990, pp. 26, 47-49)); así pues, donde Parsons encuentra una cuantificación implícita, yo encuentro una presuposición. Su posición, interesante desde el punto de vista puramente formal, no encaja, sin embargo, con la
} 
identificar los sucesos a los que se refieren sin apelar a un componente adicional, la cláusula que menciona al verbo y al sujeto de la acción. Por la conexión entre hecho y suceso, una fórmula que expresa un hecho sólo puede ser interpretada satisfactoriamente cuando se hace explícito el sujeto del verbo de la oración de acción correspondiente a la fórmula. Decir cuál es el suceso al que nos referimos pasa por decir de quién es la acción que ese suceso pretende elucidar. Por ende, la identidad de la ontología de hechos y sucesos presupone la ontología de personas. Así pues, la segunda consecuencia de mi crítica es ontológica: si en la interpretación de las oraciones de acción la ontología de sucesos requiere de alguna forma la ontología de personas - y creo que esa es una forma legítima de leer el resultado anterior-, entonces en el discurso de acción los sucesos son, en un sentido preciso, entidades derivadas de las acciones y las personas, pero no al contrario.

¿De dónde provienen estas dificultades? Si las cuantificaciones existenciales con preposiciones no expresan proposiciones completas ni tienen condiciones de verdad por deficiencias en la predicación ¿por qué sucede esto? No tengo una respuesta definitiva. Tal como veo la situación es posible decir que los predicados con preposiciones son mal formados porque violentan la sintaxis de las preposiciones, que son por naturaleza expresiones dependientes del verbo. Cuantificar sobre preposiciones y separar las preposiciones del verbo equivaldría a separar un componente que siempre va unido a otro sintácticamente; por ejemplo, sería como cuantificar la conjunción como si fuera una variable proposicional independiente cuyo significado se estableciera independi-

tesis de Davidson acerca de la perfecta simetría entre nuestro discurso sobre objetos en términos de eventos y nuestro discurso de eventos en términos de objetos porque, a pesar de que Parsons afirma que las cuestiones de identidad son secundarias para la forma lógica, el propio Davidson insiste en varios pasajes acerca de la necesidad de un criterio adecuado de identidad para eventos $(1969 a, 1985)$. Para una justificación detallada de mi punto de vista con respecto a la asimetría, Barrero (2011b). 
entemente de los enunciados o proposiciones conjuntadas ${ }^{7}$.

Esta violencia sintáctica trae consecuencias semánticas porque es intuitivamente claro que el significado de las preposiciones es incompleto sin el de los verbos a los que se añaden. Creo que podríamos concebir un lenguaje semejante al español con verbos y a partir de él construir o introducir preposiciones, pero no creo posible construir un lenguaje - semejante al español — de verbos a partir de preposiciones. ¿Cómo podría ser un fragmento lingüístico tal? ¿Qué interpretación tendría? ¿Qué posibles semejanzas guardaría con el español? Ese fragmento es inconcebible como análisis del español porque genera problemas que el español efectivamente hablado y aprendido no genera, a saber, no distingue niveles de significado que parecen básicos. Y, sin embargo, un análisis conjuntivo de las oraciones de acción da por sentado que no hay prioridad sintáctica ni prioridad de significado. Si el concepto de "presuposición" permite separar niveles de significado y señalar dependencias y asimetrías con respecto a la verdad y la falsedad, entonces es el tipo de concepto que debe aparecer en una discusión sobre la idoneidad de una u otra forma de análisis de las oraciones de acción.

Un objetor puede argüir, en una vena puramente davidsoniana, que una teoría del significado debe ser recursiva y que su recursividad juega un papel fundamental en el aprendizaje de un lenguaje. Los predicados con preposiciones serían admisibles como estructuras sintácticas de la lógica de primer orden y esto bastaría para garantizar su admisibilidad en el análisis de las oraciones de acción. A esta objeción contesto, en una vena pragmática, que aprender un lenguaje efectivamente hablado requiere un orden y arguyo que en el caso específico de las preposiciones y el español, el orden va de los verbos a las preposiciones como expresiones dependientes de los verbos. En este punto específico quisiera partir de y modificar un criterio propuesto por Kripke al tratar problemas de teoría de la referencia (1977, pp. 16-17): dado un fenómeno lingüístico

${ }^{7}$ He desarrollado algunas de estas tesis en otro trabajo Barrero (2011a). 
en español - el uso de las preposiciones en conexión con los verbos- y un análisis de ese fenómeno - la forma lógica de Davidson-, sostengo que el uso efectivo de las preposiciones en español es un contraejemplo para ese análisis ya que no creo posible concebir un lenguaje hipotético hablado por una comunidad (llamémoslo $\mathfrak{D}$ ) en donde se estipule que el análisis es correcto y el fenómeno surja. De hecho creo haber mostrado que en $\mathfrak{D}$ se presenta un problema que no se presenta en español: la identificación de acciones conectadas con preposiciones.

\subsection{Adverbios y análisis dimensional}

Ahora quiero señalar ciertas características de los adverbios atributivos y modificadores circunstanciales. En segundo lugar presentaré un esquema general de lo que debería ser un análisis de los adverbios de acción ${ }^{8}$.

De la misma forma que es el verbo el que determina el papel de la preposición, es el verbo el que determina el significado de muchos modificadores adverbiales. La formalización de Davidson oculta este hecho porque separa lógicamente esos elementos. Por ejemplo, si digo

2.3. "Mi vecino escribió una carta de inmediato",

la forma lógica propuesta por Davidson debe ser:

2.4. $\exists x[\operatorname{Escribió(Mi~vecino,~Una~carta,~} x) \wedge$ De inmediato $(x)]$,

que comparte una consecuencia lógica - 1.6 - con 1.7. Pero los parámetros para aplicar "De inmediato $(x)$ " en un caso no tienen mucho que ver con los parámetros para aplicarlo en otro. Por ejemplo, es verdad decir que si la carta se escribió en 15 minutos, se escribió de inmediato, mientras que si el burro murió 15 minutos después del disparo no murió

\footnotetext{
${ }^{8}$ Para una presentación completa y discusión detallada de este segundo punto Barrero (2010).
}

Manuscrito - Rev. Int. Fil., Campinas, v. 35, n. 2, p. 207-231, jul.-dez. 2012. 
de inmediato ${ }^{9}$. El predicado que incluye al verbo determina el espacio lógico del predicado que incluye la modificación adverbial. Por ejemplo, mientras la forma lógica de:

2.5. "Marta se lanzó a un pozo"

permite inferir

2.6. "Marta se lanzó",

la forma lógica de

2.7. "Marta se lanzó a una vida de perdición"

no lo permite Grice (1986, p. 23). Las modificaciones adverbiales están ancladas al verbo modificado y un análisis que separe las unas del otro oscurece sus propiedades semánticas. Lo que se requiere en un análisis completo de la forma lógica no es una lectura conjuntiva, sino una interpretación que tenga en cuenta las posibles construcciones del verbo, incluso en sus usos metafóricos. Un análisis conjuntivo oculta este tipo de diferencias de significado. Grice nos dice al respecto:

... (1) 'Violencia' no es el nombre de un rasgo común (por ejemplo) de estornudos y reclamos de mi esposa; los estornudos son violentos qua estornudos, los reclamos son violentos qua reclamos; y sólo son comparables en juegos de palabras. (2) De ahí, 'estornudar violentamente' no tiene el significado de la conjunción de 'estornudar' y 'hacer algo violentamente'; cuando más de 'estornudar' y 'hacer algo violentamente qua estornudar'. (3) Luego el 'análisis conjuntivo' de 'estornudar violentamente' falla $\mathrm{y}$, sin embargo, 'estornudar violentamente' implica lógicamente

\footnotetext{
${ }^{9}$ Para una explicación lingüística, la distinción entre adjetivos y adverbios relativos y absolutos presentada por Katz (1979, pp. 338-347).
} 
'estornudar'. (4) Por lo tanto las implicaciones lógicas problemáticas no se pueden explicar en general por el análisis davidsoniano de las oraciones de acción; si se les va a dar una explicación general, debe ser de otro tipo (Grice, 1986, 18 (Traducción mía)).

La última cita presenta un diagnóstico que, me parece, está en la dirección adecuada. La estrategia conjuntiva ha sido diseñada para capturar las consecuencias lógicas intuitivamente aceptables, pero las ha capturado inadecuadamente. Algunos componentes en la forma lógica dependen asimétricamente de otros componentes pero no en virtud de una simple "suma", como sucede explícitamente en los casos que he examinado en esta sección y en la anterior. Los predicados generados por adverbios atributivos no parecen tener condiciones de aplicación completas sin referencia al verbo que los adverbios modifican, análogamente a como los predicados generados por preposiciones no tienen condiciones de identidad plenas sin referencia a la cláusula que menciona al agente y al verbo. ¿Cómo captar la asimetría? Tal vez lo que esperamos de una forma lógica alternativa es la capacidad para clasificar esos niveles en la representación semántica. Una dirección de clasificación posible es partir de los agentes y los verbos y hacerlos el núcleo del significado, la dimensión significativa básica en las oraciones de acción. No afirmo que sea la única clasificación posible, pero es la que parece encajar mejor con los datos con los que he estado trabajando. Tal enfoque general estaría en capacidad de reconocer la necesidad de utilizar herramientas como la presuposición y otro tipo de infortunios lingüísticos. Y la idea de infortunio está estrechamente conectada con el concepto de dimensión evaluativa ${ }^{10}$.

\footnotetext{
${ }^{10} \mathrm{La}$ movida de los davidsonianos para responder estas objeciones puede ser de dos tipos. Uno puede suponer, de nuevo, que hay un parámetro implícito sobre el que se está cuantificando (los disparos, en un caso, la escritura de cartas, en otro) y que este parámetro es claro contextualmente (Parsons
} 
Para concluir esta crítica, quiero esbozar una estrategia dimensional para tratar otro caso interesante en las oraciones de acción, el de los adverbios de acción. El origen del análisis dimensional está en Austin. Tradicionalmente se ha creído que algunas palabras importantes como "real", "voluntario", "verdadero" denotan propiedades de objetos, acciones y emisiones, respectivamente. Esto quiere decir que siempre estamos hablando de los mismos objetos de formas diferentes. Pero esto no es más que una posición filosófica ampliamente discutible. No vay una propiedad denominada "realidad" que me permita agrupar todos los objetos existentes, de la misma forma que no hay un rasgo de las acciones que per se me permita calificarlas como voluntarias o involuntarias. Cada uno de esos términos determina una dimensión evaluativa que es la que corre con el peso ontológico, o, para decirlo con Austin, es "la palabra de pantalones" ("the word to wear the trousers") (1957, p. 182). En el caso de la acción, "voluntariamente" se aplica cuando ciertas condiciones de tipo físico-mecánico se cumplen. Cuando no se cumplen no tiene sentido hablar de acción. De ahí que pueda mover mi mano voluntariamente, pero no segregar jugos gástricos.

Una consecuencia de esta posición es que las propiedades de la negación

(1990, p. 45)). En ese caso, todos los adverbios atributivos tendrían una forma lógica elíptica. A esta tesis se le podría objetar prima facie que una explicación pragmática tendría a su disposición recursos más numerosos como la presuposición o las implicaturas convencionales para estos casos y no necesitaría multiplicar los parámetros.

O bien uno puede, en una vena tarskiana, utilizar un comparativo y dar la forma lógica por comparación del disparo o la escritura de la carta en cuestión con los otros disparos o cartas en términos de su velocidad (Lepore y Ludwig (2009, p. 166)). A esta tesis se le podría objetar prima facie que la forma lógica de los adverbios atributivos es incompleta y requiere una teoría de los comparativos que la sustente. Aunque no encuentro que ninguna de las dos posiciones estén claramente sugeridas por Davidson en sus textos, mi argumento no las afecta sin una reformulación considerable que demandaría mucho más espacio del que tengo. Agradezco a uno de los evaluadores del artículo por señalar esta limitación de mi crítica. 
de los adverbios no son las usuales, en tanto las entidades involucradas en ambos casos no son las mismas: de un lado tenemos acciones completas, mientras del otro tenemos hechos químicos, por ejemplo. La acción no involucra una sola forma de clasificación, sino diversas clasificaciones superpuestas. Existen, por ejemplo, una realización física, una etapa de apreciación de los hechos, una de información y planeación y otra de planeación y ejecución. En cada una de ellas los modificadores adverbiales son diferentes y apuntan a rasgos diferentes; la clasificación causal de la acción no es más que una clasificación posible (Austin (1957)). Rigurosamente hablando, no cabría aquí hablar de "dualismo", en tanto las clasificaciones posibles no se reducen a dos; son tantas como requieran los adverbios de acción y las excusas presentes en nuestra lengua.

\subsection{Acciones negativas y negación}

La idea misma de "acción negativa" está sujeta a discusión en el campo de la ética y algunos autores prefieren hablar de "omisiones" y discuten su estatus como acciones (Frankfurt (1994), Haji (1992), Clarke (2010)); en esta sección no pretendo presentar una definición de tal tipo de acciones. Más bien, creo que es posible encontrar contraejemplos de diversos tipos conectados con cierta clase de acciones que pueden producir problemas para la estrategia de la consecuencia lógica propuesta por Davidson. Dejo al lector en libertad para extraer sus propias conclusiones a partir de los ejemplos. Si digo (Grice (1961, p. 12)):

2.8. "Smith ha dejado de golpear a su esposa"

es suficientemente claro que (i) la estuvo golpeando hasta hace algún tiempo y (ii) que 2.8 no implica (i). Más bien, si (i) no fuera el caso, entonces $\mathbf{2 . 8}$ no podría ser verdadera, pero tampoco falsa. No podría ser verdadera porque si Smith no ha golpeado a su mujer, no puede dejar 
de hacerlo. No podría ser falsa porque eso implicaría que no ha dejado de golpearla, es decir, que continúa golpeándola, lo que contradice que (i) no sea el caso. Entonces, la forma lógica de ciertos verbos como "dejar de" es más compleja e incluye condiciones de uso no consideradas por la estrategia de la consecuencia lógica ${ }^{11}$. Por la conexión entre esa clase de verbos y otros como "continuar", por ejemplo, esas condiciones parecen estar presupuestas en muchos más casos de los considerados inicialmente. Decimos que al fallar esas condiciones el enunciado realizado mediante 2.8 falla al expresar una proposición o bien una proposición completa. Por analogía con la teoría de la referencia afirmaré que las oraciones de acción requieren ciertas condiciones de afirmabilidad sin las cuales la cuestión de su verdad o falsedad no se plantea. Así pues, algunas condiciones de significado de las oraciones de acción no se establecen por implicación.

En segundo lugar, tenemos el problema de la correlación entre acción, hecho y suceso. Para mis propósitos actuales no necesito una clasificación rigurosa, sino más bien un puñado de ejemplos en los que no parece obvio que la oración de acción se esté refiriendo a un suceso. Por ejemplo, verbos como "negarse", "rehusarse" o "resistirse" pueden funcionar para ilustrar mi punto de vista. Tomemos:

2.9. "Mi vecino no mató a mi burro"

y

2.10. "Mi vecino se negó a matar a mi burro".

La forma lógica de $\mathbf{2 . 9}$ es:

\footnotetext{
${ }^{11}$ Es necesario notar que algunos críticos de Davidson, como Evans (1976, pp. 71-72), han mostrado que casos como este pueden tratarse adecuadamente suponiendo que hay un parámetro temporal implícito y que 2.8 debería ser analizado así: "Smith golpeó a su esposa hasta $t$ y después de $t$ Smith no golpeó a su esposa".
} 
2.11. $\neg \exists x[$ Mató a(Mi vecino, Mi burro, $x)]$.

El problema es que la forma lógica de $\mathbf{2 . 1 0}$ no es tan clara por varias razones. Una, muy importante aunque no para mis objetivos en este artículo, es que probablemente estamos ante una emisión realizativa y su análisis requiera una teoría de actos ilocutivos. Otra es que intuitivamente consideramos que "negarse" es una acción intencional y hay dos posibles lecturas para la negación de $\mathbf{1 . 1 2}$, una proposicional y otra interna. La primera es:

2.12. $\neg$ Fue intencional por parte de mi vecino que $(\psi)$; mientras la segunda es:

\subsection{Fue intencional por parte de mi vecino que $(\neg \psi)$.}

Supongamos que 2.9 es verdadera. Consideremos ahora estas posibilidades: mi vecino no mató a mi burro y; o bien (i) nunca tuvo la intención de matarlo, nunca lo tuvo en mente (2.12) y es falso decir que se negó a hacerlo; o bien (ii) siempre tuvo la intención de hacerlo pero nunca lo consiguió, en cuyo caso también parece que no es correcto decir que se negó a hacerlo sino algo como "no se atrevió a hacerlo"; o bien (iii) tuvo la intención de no matarlo (2.13) y no lo mató, por lo que podemos afirmar que se negó a hacerlo (2.10 es verdadera). Concluimos que 2.9 implica $\mathbf{2 . 1 0}$ a través de 2.13, no directamente. De otra forma, cualquier hecho que haya sucedido y con el que mi vecino esté conectado podría satisfacer su acción de negarse a matar a mi burro, en tanto esos hechos estarían conectados con acciones suyas que no consisten en matar a mi burro i.e. en hacer algo que no es matar a mi burro. Pero con la condición de intencionalidad esta situación descabellada se descarta porque la acción de negarse consiste en hacer que algo en particular no suceda. Y la idea misma de "hacer que algo no suceda" es extraña en el marco conceptual de los sucesos porque tanto la existencia como inexistencia de sucesos referidos en la oración 
de acción 2.10 no bastan. Parece que lo que realmente necesitamos es especificarlas teniendo como telón de fondo el objetivo de un agente, es decir, el objeto intencional de una acción. Esa manera puede ser, como he intentado poner de manifiesto, mostrar que $\mathbf{2 . 1 0}$ y $\mathbf{2 . 1 3}$ implican 2.10.

Pero, si este análisis es correcto, "negarse" no tiene una variable de suceso característica y, a pesar de ser una acción, no es en absoluto claro cómo podríamos caracterizarla así en la propuesta de Davidson. En efecto, la forma lógica de $\mathbf{2 . 1 0}$ incluye una sola variable para suceso, la que se refiere a "matar", pero no una que se refiera a "negarse". Si la propia variable no aparece, hay acciones que no pueden considerarse como hechos y ésta es una consecuencia negativa para la tesis general acerca de la relación entre acciones, hechos y sucesos.

Si la variable aparece, hay una cuantificación sobre sucesos con otra cuantificación sobre sucesos bajo su alcance, pero no ha surgido en nuestro análisis y en ese caso podemos hacernos algunas preguntas acerca de la — supuesta - cuantificación externa: ¿cuál es su rango de aplicación? ¿Cuáles son sus condiciones de identidad? ¿Es "negarse" el mismo tipo de acción que "matar"? Parece que no: "negarse", como "dejar de", puede aplicarse a acciones enteras , "matar" no; la primera acción depende de otras acciones en un sentido en que la segunda no. Pero, si no son acciones del mismo tipo, ¿por qué deberían tener la misma forma lógica las oraciones en que aparecen los dos verbos? Lo que me parece indicar este defecto es un cortocircuito entre las oraciones de acción, de un lado y los hechos y sucesos, del otro. Concluyo a partir de este ejemplo que al menos es dudoso que la cuantificación sobre sucesos cubra todas las acciones ${ }^{12}$.

${ }^{12}$ No parece haber acuerdo entre los davidsonianos con respecto a este punto. Parsons, por ejemplo, denomina "subject-oriented modifiers" adverbios como "intencionalmente" y da una lectura semejante a la que se cita en el texto. Este caso no hace parte de lo que él denomina "Subatomic Semantics" porque no afecta a las frases o sintagmas nominales y por 


\section{CONCLUSIONES}

Creo que ante una crítica sobre una forma lógica siempre será posible contestar con otra forma lógica más compleja. No puedo ni estoy interesado en asegurar que esto no vaya a suceder en el caso de las oraciones de acción y reconozco que no he propuesto una explicación de las inferencias intuitivas a partir de oraciones de acción que motivaron a Davidson a postular una determinada estructura formal subyacente. He intentado mostrar que la verdad y la consecuencia lógica pueden ser nociones derivadas en el análisis de las oraciones de acción y que el papel principal deberían jugarlo ideas como "predicación" y "referencia" a partir de las cuales se podrían definir o al menos explicitar la verdad y las consecuencias lógicas. En otro trabajo he explorado la ruta alternativa de Montague que usa una lógica de segundo orden como herramienta formal (Barrero (2011b)). Aunque técnicamente más elegante, ese camino genera otros problemas con respecto a la identidad de los hechos y la identidad de las acciones. Quizá debamos preguntarnos si las distinciones señaladas por los adverbios, las relaciones entre acciones y movimientos a través de las preposiciones y las acciones negativas indican una dirección diferente a la ontología de sucesos. Y entonces la cuantificación sobre sucesos no es la única reconstrucción racional posible, y una ontología básica de agentes y acciones que permita construir hechos como entidades teóricas secundarias podría ser una opción atractiva, aunque su formulación y defensa exceden los límites de este artículo.

ende lo deja de lado (Parsons (1990, p. 64)). Lepore y Ludwig (2009, pp. 183-183) siguen una sugerencia de Davidson en el sentido de que "tener la intención" es un tipo de acción y proponen una lectura que usa una forma lógica como la siguiente $\exists x[\neg$ Mató a(Mi vecino, Mi burro, $x) \wedge$ Mi vecino tuvo la intención de que $\neg$ Mató a(Mi vecino, $\quad$ Mi burro, $x$ )]. Agradezco a uno de los evaluadores del artículo por llamar mi atención sobre estos aspectos. 


\section{REFERENCES}

AUSTIN, J. "Un alegato en pro de las excusas", 1957. En: Austin (1975), pp. 169-192.

— Ensayos filosóficos. Traducción de Alfonso García Suárez. Madrid: Revista de Occidente, 1975.

BARRERO, T. "Razón, acción y debilidad de la voluntad. Una lectura semántica". Ideas y Valores, No 143, pp. 161-187, 2009.

—_. "Acción y construcción lógica" (en proceso de publicación), 2011a.

—_. "Acción, identidad y eventos" (en proceso de publicación), $2011 b$.

BARWISE, J.; PERRY, J. "Situaciones y Actitudes". Traducción de J Daniel Quesada, 1981. En Valdés (1995), pp. 354-560.

CLARKE, R. "Intentional Omissions". Nô̂s, 44 (1), pp. 158-177, 2010.

DAVIDSON, D. De la verdad y de la interpretación. Traducción de Guido Filippi. Barcelona: Gedisa, 1995a.

—_ "La forma lógica de las oraciones de acción", 1967. En Davidson (1995b), pp. 133-187.

—. "Fiel a los hechos", 1969. En Davidson (1995a), pp. 57-72.

——. "Tener la intención”, 1974. En Davidson (1995b) pp. 107-119.

—_. "Realidad sin referencia", 1977. En Davidson (1995a), pp. 219228 .

——. "La individuación de los sucesos", 1969a. En Davidson (1995b), pp. 207-229. 
. Ensayos sobre acciones y sucesos. Traducción de Olbeth Hansberg, José Antonio Robles y Margarita Valdés. México-Barcelona: UNAM-Crítica, 1995b.

- Inquiries into Truth and Interpretation. Oxford: Clarendon Press, 2001.

—_. "Reply to Quine on Events", 1985. En Davidson (2002), pp. 305311.

—. Essays on Actions and Events. Oxford: Clarendon Press, 2002.

EVANS, G. "Estructura semántica y forma lógica", 1976. En Evans (1996), pp. 63-90.

—. Ensayos filosóficos. Mexico: UNAM, 1996.

FRANKFURT, H. "An Alleged Asymmetry Between Actions and Omissions". Ethics, 104 (3), pp. 620-623, 1994.

FRENCH, P.; UEHLING, T.; WetTSTEIN, H. Contemporary Perspectives in the Philosophy of Language. University of Minnesota Press: Minneapolis, 1977.

GRICE, P. "La teoría causal de la percepción". Traducción de Álvaro Caso. Cuadernos de Crítica, 41, 1961.

—_. "Actions and Events". Pacific Philosophical Quarterly, 67 (1), pp. 1-35, 1986.

HAJI, I. "A Riddle regarding Omissions". Canadian Journal of Philosophy, 22 (4), pp. 485-502, 1992.

KATZ, J. Teoría Semántica. Traducción de Juan García Puente. Madrid: Aguilar, 1972.

KRIPKE, S. Speaker's Reference and Semantic Reference. En French, Uehling, Wettstein (1977), pp. 6-27. 
LEPORE, E.; LUDWIG, K. Donald Davidson's Truth Theoretic Semantics. Oxford: Clarendon Press, 2009.

MONTAGUE, R. "Sobre la naturaleza de ciertas entidades filosófica". En Montague (1977), pp. 118-157.

— Ensayos de filosofía formal. Richmond H. Thomason (ed). Traducción de J Daniel. Quesada. Madrid: Alianza, 1977.

PARSONS, T. Events in the Semantics of English. A study in Subatomic Semantics. Cambridge, Massachusetts-Londres: The MIT Press, 1990.

TARSKI, A. "La concepción semántica de la verdad y los fundamentos de la semántica", 1944. Traducción de Mario Bunge. En Valdés (1995), pp. 275-313.

VALDÉS, L. (ed.) La búsqueda del significado. Tecnos-Universidad de Murcia: Madrid, 1995. 